\title{
Fenómenos fisiopatológicos sistémicos asociados al bruxismo de sueño
}

\section{Systemic pathophysiological phenomena associated with sleep bruxism}

Iturriaga $\mathrm{V}^{*}$, Bornhardt $\mathrm{T}^{*}$, Casassus $\mathrm{R}^{* *}$, Alveal $\mathrm{C}^{* * *}$, Medina $\mathrm{H}^{* * *}$, Reuque $\mathrm{C}^{* * *}$

\section{RESUMEN}

El bruxismo de sueño (BS) se define como un trastorno del movimiento estereotipado que se presenta durante el sueño, caracterizado por rechinamiento y/o apretamiento dentario. Se encuentra dentro de la clasificación internacional de desórdenes de sueño como un trastorno del movimiento asociado a una exagerada actividad muscular masticatoria rítmica. Se reporta una incidencia del $15 \%$ al $22 \%$ en la población general. Antiguamente se creía que la etiología del BS estaba relacionada con alteraciones oclusales; sin embargo actualmente se piensa que la causa principal está más bien relacionada con alteraciones de sueño, enfermedades del sistema nervioso central, drogas, dolor crónico, alteraciones emocionales, entre otros factores que participarían en su origen. Se ha identificado la presencia de diversos fenómenos fisiopatológicos sistémicos que acontecen durante un episodio de BS, entre los cuales encontramos los microdespertares corticales, alteraciones en el ritmo cardíaco, el tono simpático, la presión arterial, entre otros, sobre los cuales trata esta revisión.

Palabras clave: Bruxismo de sueño, bruxismo, desorden de sueño.

\section{SUMMARY}

Sleep Bruxism (BS) is defined as a stereotyped movement disorder that occurs during sleep, characterized by grinding and / or clenching tooth. It lies within the international classification of sleep disorders as a movement disorder associated with an exaggerated rhythmic masticatory muscle activity. We report an incidence of $15 \%$ to $22 \%$ in the general population. Formerly it was believed that the etiology of BS was related to occlusal alterations, but it is now thought that the main cause is more related to sleep disorders, central nervous system diseases, drugs, chronic pain, emotional distress, among other factors participate in origin. It has identified the presence of various systemic pathophysiological phenomena that occur during an episode of BS, among which are the arousal, changes in heart rate, sympathetic tone, blood pressure, among others, on which is this review.

Key words: Sleep bruxism, bruxism, sleep disorder.

Fecha de recepción: 26 de marzo de 2014.

Aceptado para publicación: 10 de mayo de 2014.

* DDS, MSC. Especialista en Trastornos Temporomandibulares y Dolor Orofacial. Departamento de Odontología Integral del Adulto. Facultad de Odontología. Universidad de La Frontera. Temuco-Chile.

** DDS, MSc, FICD. Especialista en Dolor Orofacial. Facultad de Medicina, Universidad del DesarrolloClínica Alemana. Santiago-Chile.

*** Estudiante Odontología. Escuela de Odontología. Facultad de Odontología. Universidad de La Frontera. Temuco-Chile.

Iturriaga V; Bornhardt T, Alveal C, Medina H, Reuque C. Fenómenos fisiopatológicos sistémicos asociados al bruxismo de sueño. Av. Odontoestomatol 2014; 30 (6): 325-330. 


\section{INTRODUCCIÓN}

El bruxismo de sueño (BS) se define como un trastorno del movimiento estereotipado que se presenta durante el sueño, caracterizado por rechinamiento dentario y/o apretamiento (1). Se encuentra dentro de la clasificación internacional de desórdenes de sueño (International Classification Sep Disorders ICSD, 2005) definido como un trastorno del movimiento asociado a una exagerada actividad muscular masticatoria rítmica (AMMR) principalmente del músculo masetero, con presencia de microdespertares corticales $(2,3)$ y que puede ocurrir a cualquier edad con una incidencia del 15 al $22 \%$ en la población general (4).

Antiguamente se creía que la etiología del BS estaba relacionada con alteraciones oclusales; sin embargo actualmente se piensa que la causa principal está más bien relacionada con alteraciones de sueño, enfermedades del sistema nervioso central, drogas, dolor crónico, alteraciones emocionales, entre otros factores que participarían en su origen (5). Clínicamente, el BS se diagnostica siguiendo los reportes de rechinamiento dentario por parte del compañero de cama o los padres; evaluando la presencia actual y frecuencia del rechinamiento dentario, siendo éste el criterio más decisivo; la presencia de desgastes de los dientes; y la presencia de hipertrofia de músculos de la mandíbula (6). La conciencia de este comportamiento, señalado por un miembro de la familia es reportada en el $8 \%$ de la población (7).

El diagnóstico en un laboratorio de sueño se realiza mediante un examen denominado polisomnografía, el cual permite distinguir el BS de otras actividades oromandibulares durante el sueño, como por ejemplo mioclonías oromandibulares; la acción de tragar, toser o gruñir; contactos dentarios; vocalizaciones; entre otros que pueden representar hasta el $30 \%$ de todas las actividades orales durante el sueño $(8,9)$. En una polisomnografía, se diagnostica BS si, al menos, se producen dos episodios electromiográfi$\cos (E M G)$ por noche asociados a rechinamiento dentario (8). Episodios de contracciones musculares mandibulares de más de cuatro eventos EMG por hora de sueño se consideran moderados o graves (8).
El BS se puede clasificar según los tipos de señales EMG que presente en 3 tipos:

1. Episodios fásicos o rítmicos, reconocidos si se observan al menos 3 episodios breves en EMG y están separados por 2 intervalos entre ellos.

2. Episodios tónicos, actividad sostenida que se considera si el episodio es mayor a 2 segundos.

3. Episodios mixtos, en el caso que se presente una combinación de los patrones fásico y tónico.

Aproximadamente, el 90\% de los eventos relacionados con la EMG-BS-Rechinamiento dentario en sujetos jóvenes se componen de episodios de tipo fásicos y mixtos y se ha sugerido que el patrón EMG más típico relacionado con BS se denomine actividad muscular masticatoria rítmica (AMMR). En sujetos controles la AMMR se produce durante el sueño aproximadamente una vez por hora en comparación con 2-12 veces por hora en pacientes con BS. Es importante mencionar que la AMMR relacionada al sueño también se observan en sujetos que no rechinan los dientes y su génesis probablemente estaría relacionada con una reducción de la permeabilidad de las vías respiratorias y/o lubricación baja o ausente de los tejidos de la boca y esófago (7).

Por otro lado, la mayoría de los episodios bruxísticos se producen durante el sueño No-REM, siendo frecuentemente asociado a transiciones de sueño NoREM a REM lo que sugiere que un mecanismo relacionado con la transición en estas etapas ejerce una influencia sobre las neuronas motoras que facilitarían la aparición de BS (7). Se ha identificado la presencia de diversos fenómenos fisiopatológicos sistémicos que acontecen durante un episodio de BS, entre los cuales encontramos los microdespertares corticales, el ritmo cardíaco, el tono simpático, la presión arterial, entre otros, los que se revisarán a continuación.

\section{MICRODESPERTARES CORTICALES}

Es posible que la aparición repentina de AMMR durante el sueño ocurra en ventanas de tiempo breve en el cual el cerebro pasa desde el sueño a un estado excitado. Estos períodos son denominados microdespertares corticales (MDC), definiéndose como cambios bruscos de tres a quince segundos en la actividad electroencefalográfica (EEG) acompañada de un 
Iturriaga V, Bornhardt T, Casassus R, Alveal C, Medina H, Reuque C.

aumento en el ritmo cardíaco y del tono muscular (10). Los MDC tienden a repetirse de 8 a 15 veces por hora de sueño en sujetos jóvenes sanos $(11,12)$.

Se ha demostrado que los episodios de AMMR son secundarios a una secuencia de los acontecimientos en relación con los MDC durante el sueño. Minutos previos al episodio de bruxismo en el corazón se produce un aumento de la actividad simpática autonómica, y segundos antes en el cerebro se produce una activación EEG, seguido de la aparición de actividad en los músculos suprahioideos y finalmente un movimiento de cierre con activación de los músculos masetero y/o temporal (1). En un estudio, AMMR relacionados con MDC inducidos experimentalmente fueron seguidos por rechinamiento dentario en más del $70 \%$ de los ensayos en pacientes con BS, lo que fue estadísticamente diferente con los pacientes de control (13).

\section{RITMOS CARDÍACO}

Se ha observado en relación a los episodios de BS que una aceleración en el ritmo cardíaco es una señal de un MDC. Aunque el aumento de la frecuencia cardíaca asociada con BS es importante, no es específica, ya que un aumento similar $(\approx 25 \%)$ se observa también con episodios de AMMR en individuos sin BS (14). Sin embargo, en comparación con los controles, pacientes con BS muestran un inicio más rápido del aumento de la frecuencia cardíaca.

Aunque el $90 \%$ de los episodios de AMMR y BS se han observado en asociación con actividad EEG y EMG relacionada con MDC, una secuencia clara de activación fisiológica se produce antes de la aparición del $80 \%$ de estos episodios. En el período de cuatro segundos antes de la AMMR, hay, primero, un claro incremento en la potencia de la actividad EEG seguido por un aumento en la frecuencia cardíaca. Una vez más, este aumento en la frecuencia cardíaca no es única para AMMR y BS, ya que se ha observado también con otra manifestación motora periódica durante el sueño, como son los movimientos periódicos de las extremidades durante el sueño (MPES). La evidencia anterior sugiere que los episodios de BS están estrechamente relacionados con la activación transitoria cardíaca, EEG y EMG $(15,16)$.

\section{TONO SIMPÁTICO MUSCULAR}

Se ha relacionado un elevado tono simpático general durante la vigilia con individuos que presentan BS (17). Los episodios de AMMR/BS están asociados con el movimiento corporal y también con la excitación cortical $(14,18-20)$, más específicamente, se ha demostrado que una gran proporción de episodios AMMR/BS (87,3\%) ocurren en la fase A del patrón cíclico alterno (CAP) (19). El CAP es un patrón de excitación EEG donde su fase A está vinculada una mayor actividad EEG, muscular y autonómica (21-23).

\section{PRESIÓN ARTERIAL}

Los eventos AMMR/BS están asociados con una aumento significativo de las presiones arteriales sistólica (PAS) y diastólica (PAD). Este aumento es significativo para AMMR/BS que se presentan con excitación cortical, con movimientos del cuerpo o con ambas. Estos resultados son congruentes con los cambios autonómicos actualmente conocidos que preceden a $\mathrm{AMMR} / \mathrm{BS}$, particularmente el aumento en la actividad simpática seguida por el aumento en la actividad EEG y la frecuencia cardíaca, así como también los cambios respiratorios $(14,18,19,24)$. AMMR/BS parece formar parte de la cadena de respuesta autonómica, sin embargo no se conoce la dirección causa/efecto entre ellas, además la PAS durante vigilia es significativamente mayor en el grupo de individuos con BS comparado con el grupo sin BS (25).

Otro hallazgo interesante consiste en que la PAD y PAS no siguen el mismo patrón fisiológico en asociación con AMMR/BS. La PAD vuelve a valores basales dentro de los 25 latidos tras el inicio de AMMR/ $\mathrm{BS}$; en contraste, la PAS no regresa a los valores basales tras su peak dentro de la misma ventana temporal (25).

\section{CATECOLAMINAS}

Las catecolaminas son sustancias neurotrópicas que intervienen en la actividad rítmica de los músculos masticatorios, principalmente durante el sueño (26). 
La evidencia apunta a la participación de dopamina, adrenalina, noradrenalina y serotonina en la etiología y modulación de BS (26-30).

En un estudio realizado por Seraidarian et al. (2008), se evaluaron los niveles urinarios de catecolaminas en individuos con y sin BS encontrando que estos niveles fueron significativamente mayores en el grupo con BS. Los niveles urinarios de referencia son inferiores a $60 \mu \mathrm{g} / 24 \mathrm{~h}$ de adrenalina, $200 \mu \mathrm{g} / 24 \mathrm{hr}$ para noradrenalina, y entre 65 y $400 \mu \mathrm{g} / 24 \mathrm{~h}$ para dopamina (31). Es destacable señalar que en dicho estudio, todas las personas que sufren de BS presentaron mayores niveles de adrenalina y noradrenalina, y sólo tres de los sujetos tenían niveles de dopamina dentro de los parámetros normales. En contraste, todos los sujetos de control mostraron niveles urinarios normales de todas las catecolaminas (5).

\section{DISCUSIÓN Y CONCLUSIONES}

Se ha demostrado que la actividad simpática permanece por sobre los valores iniciales durante 28 minutos tras el evento AMMR/BS (18). También se ha informado que PAS sigue siendo significativamente superior al valor inicial durante 40 segundos después de una excitación cortical (32). Por lo tanto es posible que la activación simpática con excitación durante el sueño y AMMR/BS pueda afectar variablemente las respuestas cardíaca y vascular, con una rápida normalización de la resistencia vascular periférica tal como se refleja por una rápida normalización de la PAD, y un efecto más sostenido sobre la contractilidad cardíaca conduce a la normalización retardada de la PAS. Sin embargo, en la actualidad estas hipótesis siguen siendo especulaciones que requieren mayor investigación (25).

Se ha visto que es posible evitar la excesiva activación cardíaca simpática asociada a episodios de BS por el uso de clonidina, agonista alfa-adrenérgico. La administración de clonidina antes de dormir reduce la actividad simpática cardíaca y el número de episodios de BS en un $60 \%$ (18). Sin embrago, cabe destacar las reacciones adversas que produce este fármaco, tales como bradicardia, somnolencia (33), sedación e hipotensión (34).
Específicamente, los MDC están normalmente caracterizados por taquicardia, a menudo seguidos por bradicardia (35), y pueden ocurrir con o sin desincronización EEG (excitación cortical o subcortical). Estos eventos son similares a las secuencias fisiopatológicas involucradas en los episodios AMMR/BS, los cuales consisten en las siguientes actividades:

1. Incremento en la actividad simpática previo al inicio de AMMR/BS 4 a 8 minutos antes (18).

2. Aumento en la actividad EEG (excitación cortical) 4 segundos antes.

3. Taquicardia, que ocurre 1 segundo antes de AMMR/BS $(14,19)$.

4. Aumento en la amplitud respiratoria coincidente con el inicio de AMMR/BS (24).

Por lo dicho anteriormente, queda en evidencia que en un episodio de bruxismo de sueño no sólo se produce un rechinamiento dentario con actividad de los músculos depresores y elevadores mandibulares, sino que se produce una cascada de acontecimientos donde participan el ritmo cardíaco, el sistema cardíaco autonómico, el tono simpático muscular, la presión arterial, algunas catecolaminas, entre otros. Por lo que es necesario dejar de ver el bruxismo como una entidad oromandibular aislada y comenzar a mirarlo como una reacción corporal sistémica, tanto en su diagnóstico como en su tratamiento.

\section{BIBLIOGRAFÍA}

1. Lavigne GJ, Huynh N, Kato T, Okura K, Adachi K, Yao D, et al. Genesis of sleep bruxism: Motor and autonomic-cardiac interactions. Arch Oral Biol 2007;52:381-4.

2. Kato T, Masuda Y, Yoshida A, Morimoto T. Masseter EMG activity during sleep and sleep bruxism. Arch ItalBiol2011;149:478-91.

3. Lavigne GJ, Rompre PH, Poirier G, Huard H, Kato T, Montplaisir JY. Rhythmic masticatory muscle activity during sleep in humans. J Dent Res 2001;80:443-8.

4. Attanasio R. Nocturnal bruxism and its clinical management. Dent Clin North Am 1991;35:24552. 
5. Seraidarian P, Seraidarian PI, das Neves Cavalcanti B, Marchini L, Claro Neves AC. Urinary levels of catecholamines among individual with and without sleep bruxism. Sleep Breath 2009; 13:85-8.

6. Lavigne GJ, Manzini C, Kato T. Sleep bruxism. EnKryger $\mathrm{MH}$, Roth $\mathrm{T}$, Dement WC, eds. Principles and Practice of Sleep Medicine. Elsevier Saunders: Philadelphia; 2005.

7. Lavigne GJ, Khoury S, Abe S, Yamaguchi T, Raphael K. Bruxism physiology and pathology: an overview for clinicians. J Oral Rehabil 2008;35:476-94.

8. Lavigne GJ, Rompre PH, Montplaisir JY. Sleep bruxism: validity of clinical research diagnostic criteria in a controlled polysomnographic study. J Dent Res 1996;75:546-52.

9. Kato T, Montplaisir JY, Blanchet PJ, Lund JP, Lavigne GJ. Idiopathic myoclonus in the oromandibular region during sleep: a possible source of confusion in sleep bruxism diagnosis. MovDisord 1999; 14:865-71.

10. Lobbezoo F, Lavigne G. Do Bruxism and Temporomandibular Disorders Have a Cause-and-Effect Relationship? J Orofac Pain 1997;11:15-23.

11. Parrino L, Smerieri A, Rossi M, Terzano MG. Relationship of slow and rapid EEG components of CAP to ASDA arousals in normal sleep. Sleep 2001;24:881-5.

12. Boselli M, Parrino L, Smerieri A, Terzano MG. Effect of age on EEG arousals in normal sleep. Sleep 1998;21:351-7.

13. Kato T, Montplaisir JY, Guitard F, Sessle BJ, Lund JP, Lavigne GJ. Evidence that experimentally induced sleep bruxism is a consequence of transient arousal. J Dent Res 2003;82:284-8.

14. Kato T, Rompre P, Montplaisir JY, Sessle BJ, Lavigne GJ. Sleep bruxism: an oromotor activity secondary to micro-arousal. J Dent Res 2001;80: 1940-4.
15. Bader G, Lavigne G. Sleep bruxism; an overview of an oromandibular sleep movement disorder. Sleep Med 2000;4:27-43.

16. Lavigne GJ, Manzini C. Sleep bruxism and concomitant motor activity. En Principles and Practice of Sleep Medicine. Kryger MH, Roth T, Dement WC, eds. Philadelphia: Elsevier Saunders; 2000.

17. Marthol H, Reich S, Jacke J, Lechner KH, Wichmann M, Hilz MJ. Enhanced sympathetic cardiac modulation in bruxism patients. Clin Auton Res 2006;16(4):276-80.

18. Huynh N, Kato T, Rompré PH, Okura K, Saber $M$, Lanfranchi PA, et al. Sleep bruxism is associated to micro-arousals and an increase in cardiac sympathetic activity. J Sleep Res. 2006; $16: 276-80$

19. Macaluso GM, Guerra P, Di Giovanni G, Boselli M, Parrino L, Terzano MG. Sleep bruxism is a disorder related to periodic arousals during sleep. J Dent Res 1998;77:565-73.

20. Kato T, Montplaisir JY, Guitard F, Sessle BJ, Lund JP, Lavigne GJ. Evidence that experimentally induced sleep bruxism is a consequence of transient arousal. J Dent Res 2003;82:284-8.

21. Terzano MG, Parrino L, Boselli M, Smerieri A, Spaggiari MC. CAP component and EEG symchronization in the first 3 sleep cycles. Clin Neurophysiol 2000;111:283-90.

22. Terzano GM, Parrino L, Rosa A, Palomba V, Smerieri A. CAP and arousals in the structural development of sleep: an integrative perspective. Sleep Med 2002;3:221-9.

23. Carra MC, Macaluso GM, Rompré PH, Huynh N, Parrino L, Terzano MG, et al. Clonidine has a paradoxical effect on cyclic arousal and sleep bruxism during NREM sleep. Sleep 2010;33: 1711-6.

24. Khoury S, Rouleau GA, Rompre PH, Mayer P, Montplaisir J, Lavigne GJ. A significant increase 
in breathing amplitude precedes sleep bruxism. Chest 2008;134:332-7.

25. Nashed A, Lanfranchi P, Rompré P, Carra MC, Mayer P, Colombo R, Huynh N, et al. Sleep bruxism is associated with a rise in arterial blood pressure. Sleep 2012;35:529-36.

26. Lobbezoo F, Lavigne GJ, Tanguay R, Montplaisir JY. The effect of catecholamine precursor L-Dopa on sleep bruxism: a controlled clinical trial. Mov Disord 1997;12:73-8.

27. Lavigne GJ, Rompré PH, Poirierl G, Huard H, Katol T, Montplaisir JY. Rhythmic masticatory muscle activity during sleep in humans. J Dent Res 2001;80:443-8.

28. Lobbezoo F, Soucy JP, Hartman NG, Montplaisir JY, Lavigne GJ. Effects of the D2 receptor agonist bromocriptine on sleep bruxism: report of two single patient trials. J Dent Res 1997;76:1610-4.

29. Chen WH, Lu YC, Lui CC, Liu JS. A proposed mechanism for diurnal/nocturnal bruxism: hypersensitivity of presynaptic dopamine receptors in the frontal lobe. J Clin Neurosci 2005;12:161-3.

30. Magee KR. Bruxism related to levodopa therapy. JAMA 1970;214:147.

31. Ross GA, Newbould EC, Thomas J, Bouloux PM, Besser GM, Perrett D, et al. Plasma and 24 hurinary catecholamine concentrations in normal and patient populations. Ann Clin Biochem 1993;30:38-44.

32. Blasi A, Jo J, Valladares E, Morgan BJ, Skatrud JB, Khoo MC. Cardiovascular variability after arousal from sleep: time-varying spectral analysis. J Appl Physiol 2003;95:1394-404.

33. Daviss WB, Patel NC, Robb AS, McDermott MP, Bukstein OG, Pelham WE Jr, et al. Clonidine for attention-deficit/hyperactivity disorder: II. ECG changes and adverse events analysis. J Am Acad Child Adolesc Psychiatry 2008;47:189-98.

34. Marinangeli F, Ciccozzi A, Donatelli F, Di Pietro A, Iovinelli G, Rawal N, et al. Clonidine for treatment of postoperative pain: a dose-finding study. Eur $\mathrm{J}$ Pain 2002;6:35-42.

35. Sforza E, Jouny C, Ibañez V. Cardiac activation during arousal in humans: further evidence for hierarchy in the arousal response. Clin Neurophysiol 2000;111:1611-9.

\section{CORRESPONDENCIA}

Verónica Iturriaga

Claro Solar 115

Departamento de Odontología Integral del Adulto Facultad de Odontología. Universidad de La Frontera. Temuco-Chile.

Correo electrónico: veronica.iturriaga@ufrontera.cl 